\title{
Simultaneous occurrence of Hodgkin's lymphoma and multiple myeloma: A case report and review of the literature
}

\author{
CHEN HUANG $^{1}$, GUIMIN ZHAO ${ }^{1}$, LIANJING WANG ${ }^{1}$, HUICHAO ZHANG $^{2}$, XIAOLIN WU ${ }^{1}$, \\ MINGZENG ZHANG ${ }^{1}$, RUIJUAN MA ${ }^{1}$, LING WANG ${ }^{1}$, YUEPING LIU ${ }^{3}$ and LIHONG LIU ${ }^{1}$ \\ Departments of ${ }^{1}$ Hematology, ${ }^{2}$ Inspection and ${ }^{3}$ Pathology, The Fourth Hospital of Hebei Medical University, \\ Shijiazhuang, Hebei 050011, P.R. China
}

Received February 5, 2015; Accepted March 4, 2016

DOI: $10.3892 / 01.2016 .4503$

\begin{abstract}
Hodgkin's lymphoma (HL) is a type of hematological neoplasm that generally appears alone, with a low incidence. The majority of cases histopathologically present as B-cell lymphoma. Multiple myeloma (MM) is defined as the neoplastic proliferation of a single clone of plasma cells producing a monoclonal immunoglobulin (Ig). The coexistence of HL and MM is rare, however, the present study reports such a case. On May 31, 2012, a 45-year-old man was diagnosed with HL, stage III, 31 months ago. At the same time, computed tomography and magnetic resonance imaging showed osteolytic lesions, a significant increase in $\operatorname{IgA} \lambda$ chains, and multiple myeloma cells on bone marrow aspiration. Following 8 cycles of chemotherapy, the patient received maintenance treatment with thalidomide and dexamethasone. During 2 years of follow-up, the patient has maintained a complete response for $\mathrm{HL}$ and a stable disease state for MM. The coexistence of HL and MM is rare. Further study of such cases may explain the associations between these two tumors and aid the production of effective treatment options.
\end{abstract}

\section{Introduction}

Hodgkin's lymphoma (HL) is a unique type of lymphoma that is one of the most common malignant tumors in young individuals. HL is an uncommon malignancy, with 7,000-7,500 novel cases diagnosed annually in the USA; however, the incidence of the disease in China is lower compared with the global average $(1,2)$. The majority of HL patients present with early stage disease (3). Pathology is an important means of diagnosis and classification. Primary ABVD combination chemotherapy

Correspondence to: Miss. Lihong Liu, Department of Hematology, The Fourth Hospital of Hebei Medical University, 169 Tianshan Street, Shijiazhuang, Hebei 050011, P.R. China

E-mail: ejb_007@163.com

Key words: Hodgkin's lymphoma, multiple myeloma, disease diagnosis
[25 $\mathrm{mg} / \mathrm{m}^{2}$ intravenous (i.v.) pirarubicin, $10 \mathrm{mg} / \mathrm{m}^{2}$ i.v. bleomycin, $375 \mathrm{mg} / \mathrm{m}^{2}$ i.v. dacarbazine and $1.4 \mathrm{mg} / \mathrm{m}^{2}$ i.v. vincristine; days 1 and 5], followed by involved-field irradiation is the standard treatment for patients with early-stage HL, with an overall survival (OS) $>95 \%$ (4).

Multiple myeloma (MM) is a tumor that generally effects in the elderly; it is a neoplastic plasma-cell disorder that may be distinguished by the clonal proliferation of malignant plasma cells in the bone marrow and the presence of monoclonal protein in the blood or urine (5). The disease is associated with organ dysfunction. MM accounts for $\sim 1 \%$ of neoplasms and $13 \%$ of hematological cancers. In western countries, the annual age-adjusted incidence is 5.6 cases per 100,000 population (6). Eventually, numerous complications tend to appear alongside the disease, including bone destruction, anemia, renal insufficiency, hypercalcemia and susceptibility to infection (5). In recent years, the OS and progression-free survival of MM has been increasing due to the application of targeted drugs, including bortezomib, thalidomide and lenalidomide (7). The coexistence of HL and MM is rare (8-10). The current study reports the case of a patient who presented with HL and MM at the same time, for which a good outcome was achieved using chemotherapy.

\section{Case report}

A 45-year-old man was admitted to The Fourth Hospital of Hebei Medical University (Shijiazhuang, Hebei, China) in May 2012 due to lumbago and intermittent fever that had been apparent since March 2012. The patient's temperature was $\sim 39^{\circ} \mathrm{C}$, without the symptoms of chills, coughing, abdominal pain or diarrhea. Since the symptoms first occurred, the patient had experienced no night sweats or weight loss. The patient was previously healthy with no relevant family medical history. Upon physical examination, the patient's temperature was $38.5^{\circ} \mathrm{C}$ (normal range, $36-37^{\circ} \mathrm{C}$ ), with a pulse rate of 108 beats/min (normal range, $60-100$ beats $/ \mathrm{min}$ ), a respiratory rate of 20 breaths $/ \mathrm{min}$ (normal range, 16-20 breaths/min), and a blood pressure of $134 / 90 \mathrm{mmHg}$ (normal range, $90-140 / 60-90 \mathrm{mmHg}$ ). Two lymph nodes, $\sim 1 \mathrm{~cm}$ in diameter, were palpable in the left supraclavicular region, and one lymph node, $\sim 3 \mathrm{~cm}$ in diameter, was palpable in the left armpit. The patient experienced pain upon percussion over the fourth and fifth lumbar vertebrae. 


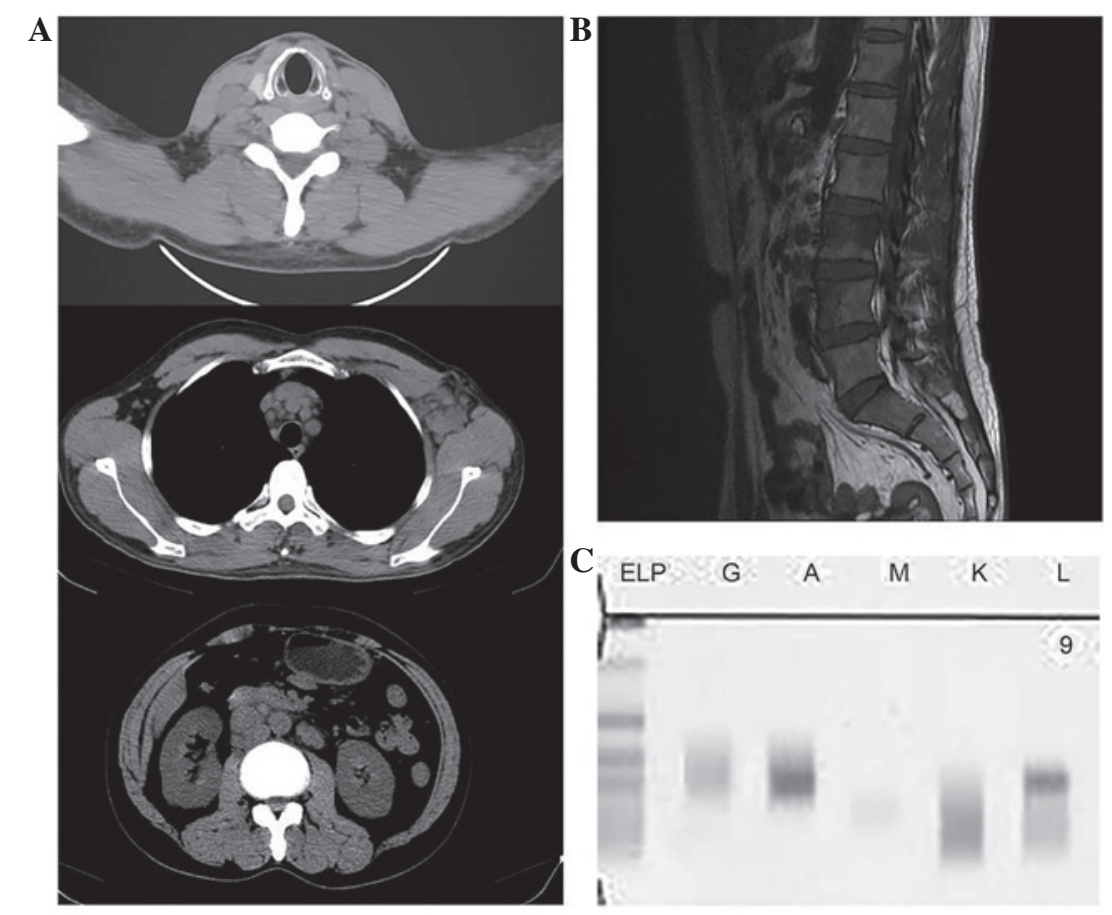

Figure 1. Whole-body computed tomography scans and lumbar magnetic resonance imaging showing (A) enlargement of the left supraclavicular, left axillary and abdominal lymph nodes, and (B) multiple low signals in the lumbar spine. (C) Serum immunofixation electrophoresis revealing a spike in immunoglobulin A $\lambda$ chain levels. G, immunoglobulin G; A, immunoglobulin A; M, immunoglobulin M; K, $\kappa$ light chain; L, $\lambda$ light chain; 9 , serial number of patient.

The complete blood count revealed a white blood cell count of $9.64 \times 10^{9} / 1$ (normal range, $4-10 \times 10^{9} / 1$ ), a hemoglobin level of $107.4 \mathrm{~g} / 1$ (normal range, $120-160 \mathrm{~g} / \mathrm{l}$ ) and a platelet count of $177.7 \times 10^{9} / 1$ (normal range, $100-300 \times 10^{9} / 1$ ), and the erythrocyte sedimentation rate was $111 \mathrm{~mm} / \mathrm{h}$ (normal range, $<15 \mathrm{~mm} / \mathrm{h}$ ). Routine blood biochemical examination revealed the following: Total serum protein, $123.1 \mathrm{~g} / 1$ (normal range, 65-85 g/l); albumin, $28.5 \mathrm{~g} / 1$ (normal range, 40-55 g/l); globulin, $40.3 \mathrm{~g} / \mathrm{l}$ (normal range, 20-40 g/l); immunoglobulin G (IgG), $14.2 \mathrm{~g} / 1$ (normal range, 8-16 g/l); IgA, $10 \mathrm{~g} / 1$ (normal range, 0.5-2.2 g/l); and $\mathrm{IgM}, 1.1 \mathrm{~g} / \mathrm{l}$ (normal range, 0.7-3.3 g/l). The lactate dehydrogenase level was 4.157 units/l (normal range, 80-250 units/l), and the $\beta 2$ microglobulin $(\beta 2-\mathrm{MG})$ and $\mathrm{C}$-reactive protein levels were 3.68 (normal range, 0.8-2.80 mg/l) and $84.4 \mathrm{mg} / \mathrm{l}$ (normal range, $0-8 \mathrm{mg} / \mathrm{l}$ ), respectively.

The routine whole-body scan showed diffuse lymph node enlargement and a small amount of fluid accumulation in the pelvic cavity (Fig. 1A). The lumbar magnetic resonance imaging showed multiple low signals in the thoracic, lumbar and sacral spine (Fig. 1B).

Serum protein electrophoresis was performed by capillary zone electrophoresis (Paragon CZE 2000; Beckman Coulter Inc., Brea, CA, USA). The antiserum of $\operatorname{IgG}, \operatorname{IgA}, \operatorname{IgM}, \kappa$ and $\lambda$ light chain proteins were tested and serum immunofixation electrophoresis demonstrated a spike in $\operatorname{IgA} \lambda$ chains (Fig. 1C).

The bone marrow aspiration showed that MM cells accounted for $20 \%$ of the nucleated cells (Fig. 2A). The lymph node and bone marrow biopsies were $4 \%$ formalin-fixed, paraffin-embedded and sectioned (4-5 $\mu \mathrm{m}$ thick) for staining. Routine hematoxylin and eosin staining and necessary immunohistochemical (IHC) staining were performed using the polymer-enhanced two-step IHC method (11). For the IHC analysis of the paraffin-embedded sections, the antibodies that were selected included: Mouse anti-human monoclonal antibodies, cluster of differentiation (CD)20 (dilution, 1:100; catalog no., Kit-0001), Ki67 (dilution, 1:200; catalog no., Kit-0005), CD4 (dilution, 1:200; catalog no., MAB-0251), CD15 (dilution, 1:200; catalog no., MAB-0015), CD21 follicular dendritic cells (dilution, 1:200; catalog no., MAB-0339), CD30 (dilution, 1:200; catalog no., MAB-0023), CD38 (dilution, 1:200; catalog no., MAB-0341), CD56 (dilution, 1:200; catalog no., Kit-0028), CD138 (dilution, 1:200; catalog no., MAB-0200), CD163 (dilution, 1:200; catalog no., MAB-0206), $\kappa$ light chain (dilution, 1:200; catalog no., MAB-0356), $\lambda$ light chain (dilution, 1:200; catalog no., MAB-0357), CD45 leucocyte common antigen (LCA) (dilution, 1:200; catalog no., Kit-0024) and melanoma associated antigen (mutated) 1 (MUM1) (dilution, 1:200; catalog no., MAB-0339); mouse anti-virus monoclonal Epstein-Barr encoded RNA (EBER) antibody (dilution, 1:200; catalog no., MAB-0339); programmed cell death 1 (dilution, 1:50; catalog no., MAB-0654) and Vs38c (dilution, 1:200; catalog no., MAB-0296) antibodies; rabbit anti-human monoclonal CD3e (dilution, 1:200; catalog no., Kit-0003) and paired box 5 (Pax5) (dilution, 1:200; catalog no., RMA-0611) antibodies; rabbit anti-human polyclonal IgM antibody (dilution, 1:200; catalog no., RAB-0103); and goat anti-human polyclonal chemokine (C-X-C motif) ligand 13 (CXCL13) antibody (dilution, 1:200; catalog no., GAB-0616). All primary antibodies were purchased from Fuzhou Maixin Biotech. Co., Ltd. (Fuzhou, China). Secondary antibodies used were from ultraView Universal DAB Detection kit (Roche Diagnostics, Mannheim, Germany). Primary antibodies were incubated for $2 \mathrm{~h}$ at $37^{\circ} \mathrm{C}$ and secondary antibodies were incubated for $24 \mathrm{~h}$ at $4^{\circ} \mathrm{C}$.

The IHC results of the bone marrow biopsy showed positive staining for CD138, CD38, Ki-67, MUM1 and the $\kappa$ and 

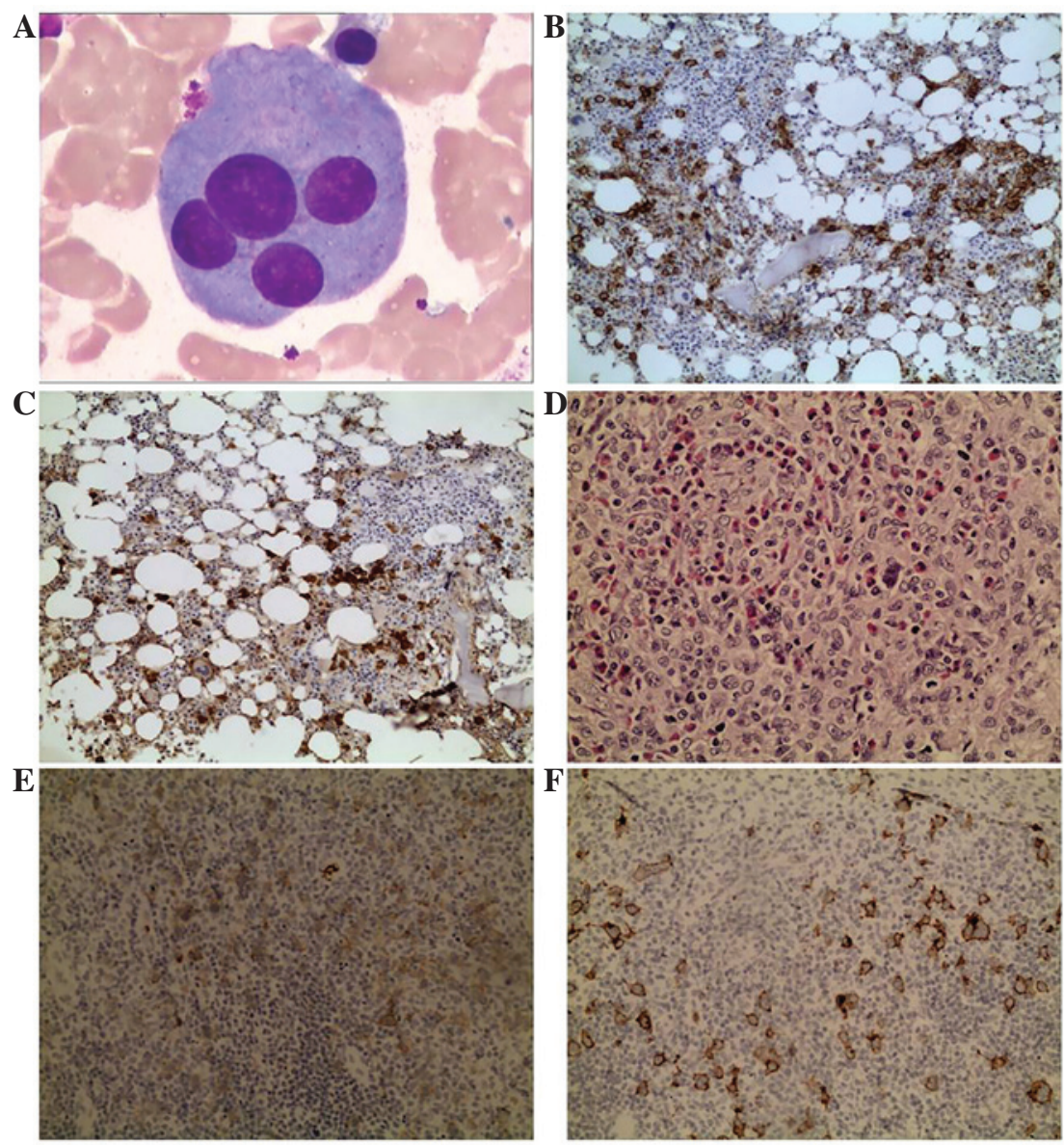

Figure 2. Bone marrow smears of the left anterior superior iliac spine showing (A) a multinucleated myeloma cell (Wright's staining; x1,000 magnification). Immunohistochemical analysis of the bone marrow biopsy showing positive staining of the cells for (B) cluster of differentiation (CD) 138 , and (C) $\lambda$ light chain (more than $\kappa$, monoclonality) (x20 magnification). Biopsy of the enlarged lymph node of the left supraclavicular region showing (D) a large number of typical Reed-Sternberg cells in the interfollicular (hematoxylin and eosin stain; $x 40$ magnification). Immunohistochemical analysis showing positive staining of the Reed-Sternberg cells for (E) CD15 and (F) CD30 (x40 magnification).

$\lambda$ light chains, and negative staining for $\mathrm{CD} 3 \varepsilon, \mathrm{CD} 30$, Pax5, CD20, EBER, CD56, IgM and Vs38c (Fig. 2B and C). Excisional lymph node biopsy showed enlarged lymph nodes with effaced nodal architecture, which was replaced by a mixed inflammatory infiltrate with scattered large-sized classical Reed-Sternberg cells (Fig. 2D). The immunohistochemical analysis showed positive staining of the cells for CD163, Pax5, CD30, CD15, CD3e, CD4, CD21 follicular dendritic cells, programmed cell death 1 and Ki-67 (40\%), and negative staining of the cells for CD20, EBER, CD45 LCA and CXCL13 (Fig. 2E and F). The patient was subsequently diagnosed with mixed cellularity HL, stage IIIB, according to the Ann Arbor System (12), combined with MM (IgA $\lambda)$, stage IIIA, according to the Durie-Salmon System (13).

The patient received 3 cycles of chemotherapy, consisting of $25 \mathrm{mg} / \mathrm{m}^{2}$ i.v. pirarubicin, $10 \mathrm{mg} / \mathrm{m}^{2}$ i.v. bleomycin, $375 \mathrm{mg} / \mathrm{m}^{2}$ i.v. dacarbazine and $1.4 \mathrm{mg} / \mathrm{m}^{2}$ i.v. vincristine on days 1 and 15, and $100 \mathrm{mg}$ oral (p.o.) thalidomide on days 1-28 (ABVDT regimen). This resulted in a complete response (CR) for the HL and partial remission for the MM. Following 3 further cycles of ABVDT chemotherapy, a CR was maintained for the HL and a stable disease (SD) state was achieved for the MM. The patient subsequently received 2 cycles of chemotherapy consisting of $0.5 \mathrm{mg}$ /day i.v. vincristine, $10 \mathrm{mg} / \mathrm{m}^{2}$ i.v. pirarubicin and
$10 \mathrm{mg} / \mathrm{m}^{2}$ i.v. dexamethasone on days $1-4$ and 9-12, and $100 \mathrm{mg}$ p.o. thalidomide on days 1-28). The patient refused autologous stem cell transplantation, but was administered maintenance treatment consisting of weekly dexamethasone $(7.5 \mathrm{mg})$ and daily thalidomide $(100 \mathrm{mg})$ tablets, with dexamethasone alternated with thalidomide every 3 months. During 2 years of follow-up, the patient has maintained a CR for the HL and a SD state for the MM, and continues to be followed up every 6 months.

\section{Discussion}

HL is a malignant tumor that is derived from the lymphoid tissues; the incidence rate of HL is 1.4-6.5 cases/100,000 individuals in China (14). In 2013, 9,000 cases of HL were diagnosed in the United States and 1,180 individuals were predicted to succumb to the disease (8). Nodular sclerosing HL is more common in the developed countries of Europe and America, while mixed cellularity HL is more common in China. Classic HL represents $\sim 10 \%$ of all lymphomas diagnosed annually in the developing world (8). MM is a clonal B-cell disorder with characteristic B-lymphocyte and plasma cell proliferation and accumulation. The annual incidence of the disease is 6 cases $/ 100,000$ individuals in Western countries, 
thus representing the second most frequently occurring hematological malignancy following non-HLs. In China, the annual incidence of $\mathrm{MM}$ is $\sim 1$ case/100,000 individuals $(9,10)$. The simultaneous occurrence of two types of lymphatic system malignant tumor within the same patient is extremely rare, with an occurrence rate of 1.4-6.5 cases/1,000,000 individuals (15).

The pathogenesis of this simultaneous occurrence are not yet clear. Previous studies have also reported the simultaneous occurrence of HL and chronic lymphocytic leukemia, peripheral T-cell lymphoma or diffuse large B-cell lymphoma (16-18). Furthermore, certain patients have developed plasmocytoma $(15,19)$, MM $(20,21)$ or monotypic plasma cell proliferations of unknown significance (22) following therapy for HL; it is unclear whether such secondary tumors are associated with the side effects of the first cancer treatment. Hasskarl et al (23) suggested that MM is associated with hematological neoplasms. A total of 589 consecutive cases of MM were reviewed, and 13 (2.2\%) were found with different hematological neoplasms. Those MM patients with prior or synchronously $(\mathrm{p} / \mathrm{s})$ different neoplasms exhibited a hazard ratio (HR) of 1.2 [95\% confidence interval (CI), 0.8-2.0) for impaired OS, whereas those with subsequent neoplasms exhibited an HR of 2.5 (95\% CI, 1.4-4.4). These results indicated that $\mathrm{p} / \mathrm{s}$ different neoplasms occur more frequently than subsequent different neoplasms, and that a worse prognosis is associated with subsequent different neoplasms. However, the simultaneous occurrence of HL and MM is extremely rare, with only a few reported cases. One of these studies from 1999 (24) described the case of a 37-year-old man who presented with a $10 \times 8-\mathrm{cm}$, right cervical tumor that was found by the patient 6 months previously. The patient experienced fatigue and a loss of appetite. Following a biopsy of the cervical lymph node, a histological diagnosis was formed of nodular sclerosing HL with a Langerhans cell focus in an affected node. Serum electrophoresis revealed an IgA $\kappa$ paraprotein level of 3,200 mg and a $\beta 2-\mathrm{MG}$ level of $7.6 \mathrm{mg} / \mathrm{l}$. Free immunoglobulin $\kappa$ light chains were detected in the urine. X-ray examination of the skeleton revealed osteolytic lesions in the ribs, the calvarium, the dorsolumbar region of the vertebral column, the pelvis and the femoral metaphyses. Computed tomography revealed enlargement of the bilateral cervical lymph nodes without enlargement of the spleen, liver or other lymph nodes. Bone marrow examination demonstrated extensive infiltration by atypical plasma cells. MM without bone marrow HL infiltration was considered as the final diagnosis, and 3 cycles of ABVD led to complete remission of the HL.

In 2000, Lalayanni et al (25) reported the case of a 52-year-old male who was diagnosed with simultaneous MM and HL in the absence of any prior treatment. Autonomous progression of plasma cell dyscrasia did not occur, but was instead associated with HL. In June 1993, the patient presented with a relapsing-remitting fever that had persisted for 3 months. Physical and laboratory findings were normal, with the exception of anemia (hemoglobin, $9 \mathrm{~g} / \mathrm{dl}$ ), an erythrocyte sedimentation rate of $102 \mathrm{~mm} / \mathrm{h}$ and the presence of an IgG- $\kappa$ monoclonal component with Bence-Jones protein in the urine. Up to $60 \%$ plasma cell infiltration of the bone marrow was determined, but a bone X-ray was normal. A diagnosis of MM was formed and the patient was administered melphalan-prednisolone. After 2 months, all the findings remained unchanged and left axillary lymphadenopathy developed. Mixed cellularity HL (stage IIB) was revealed upon biopsy. The patient achieved complete remission of each malignancy following 6 courses of cyclophosphamide, vincristine, procarbazine and prednisone/ABVD and involved-field radiotherapy. The HL (stage IIIB) relapsed 1 year later. Complete remission was achieved again for 4 months with a chlorambucil, vinblastine, procarbazine and prednisolone regimen, however, following this, relapse of the two diseases was noted. Two courses of intermediate-dose melphalan were not successful in achieving control of either disease. Infiltration of the bone marrow by Hodgkin and plasma cells was demonstrated on biopsy. These findings were more compatible with constitutional mosaicism. Another 3 courses of ABVD led to complete remission of the $\mathrm{HL}$ and MM for the third time, however, the patient succumbed to acute hepatitis B and hepatic failure.

It has been suggested that certain HL cases are of B-cell origin $(26,27)$. Malignant HL cells could be a consequence of the arrest of maturation in the B-cell series, and, by contrast, plasma cell neoplasms could be a consequence of the complete differentiation of these cells (28). A decrease in cell-mediated immunity in HL may also explain the presence of other neoplasms, such as MM, and similar abnormalities in karyotype have been found within them (29). Cytokines, such as interleukin 6 (IL-6), may be important in the formation of each disease. In addition to acting as a factor for plasma cell growth and apoptosis (30), IL-6 mRNA is also expressed in Reed-Sternberg cells (infiltrating the marrow), and high IL-6 levels are found in advanced HL (31). Although this stimulus does not appear to be sufficient enough for the promotion of monoclonality, excess IL-6 in the marrow microenvironment may be able to directly stimulate the long-living plasma cells located there (28).

The coexistence of HL and MM is rare, and the pathogenesis is unclear, with few cases reported in the literature; however, the present literature review indicates that the two tumors may be associated. The management therapy for this entity is not standardized, as studies in the literature are confined to a small number of case reports and small case series. The current study reports a rare case in which two types of low-incidence hematological neoplasm occurred simultaneously in the same patient. A B lymphocyte tumor and plasma cell neoplasm were present in the patient, which provided more information to enable the future optimization of treatment. Further study of such cases will aim to explain the associations between these two tumors and provide improved treatment selection.

\section{References}

1. Townsend W and Linch D: Hodgkin's lymphoma in adults. Lancet 380: 836-847, 2012.

2. Glaser SL and Hsu JL: Hodgkin's disease in Asians: Incidence patterns and risk factors in population-based data. Leuk Res 26: 261-269, 2002.

3. Gobbi PG, Ferreri AJ, Ponzoni M and Levis A: Hodgkin lymphoma. Crit Rev Oncol Hematol 85: 216-237, 2013.

4. Klasa RJ, Connors JM, Fairey R, Gascoyne R, Hoskins P, O'Reilly S, Shenkier T, Voss K and Wilson K; British Columbia Cancer Agency (BCCA): Treatment of early stage Hodgkin's disease: Improved outcome with brief chemotherapy and radiotherapy without staging laparotomy. Annal Oncol 7 (Suppl 3): 21, 1996.

5. Raab MS, Podar K, Breitkreutz I, Richardson PG and Anderson KC: Multiple myeloma. Lancet 374: 324-339, 2009. 
6. Palumbo A and Anderson K. Multiple myeloma: N Engl J Med 364: 1046-1060, 2011.

7. Sonneveld P, Schmidt-Wolf IG, van der Holt B, El Jarari L, Bertsch U, Salwender H, Zweegman S, Vellenga E, Broyl A, Blau IW, et al: Bortezomib induction and maintenance treatment in patients with newly diagnosed multiple myeloma: Results of the randomized phase III HOVON-65/ GMMG-HD4 trial. J Clin Oncol 30: 2946-2955, 2012.

8. Diefenbach C and Advani R: Customized targeted therapy in Hodgkin lymphoma: Hype or hope?. Hematol Oncol Clin North Am 28: 105-122, 2014.

9. Tosi P: Diagnosis and treatment of bone disease in multiple myeloma: Spotlight on spinal involvement. Scientifica (Cairo) 2013: 104546, 2013

10. Jemal A, Siegel R, Xu J and Ward E: Cancer statistics, 2010. CA Cancer J Clin 60: 277-300, 2010.

11. Sabattini E, Bisgaard K, Ascani S, Poggi S, Piccioli M, Ceccarelli C, Pieri F, Fraternali-Orcioni G and Pileri SA: The EnVision++ system: A new immunohistochemical method for diagnostics and research. Critical comparisonwith the APAAP ChemMate, CSA, LABC, and SABC techniques. J Clin Pathol 51: 506-511, 1998.

12. Durie BG, Salmon SE: A clinical staging system for multiple myeloma. Correlation of measured myeloma cell mass with presentingclinical features, response to treatment, and survival. Cancer. 36: 842-854, 1975.

13. Smithers DW: Summary of papers delivered at the Conference on Staging in Hodgkin's Disease (Ann Arbor). Cancer Res 31: 1869-1870, 1971 .

14. Yang QP, Zhang WY, Yu JB, Zhao S, Xu H, Wang WY, Bi CF, Zuo Z, Wang XQ, Huang J, et al: Subtype distribution of lymphomas in Southwest China: Analysis of 6,382 cases using WHO classification in a single institution. Diagn Pathol 6: 77 , 2011.

15. Gherlinzoni F, Cavo M, Pileri S, Rivano MT, Boriani S, Biagini R and Emiliani E: Solitary plasmocytoma of the bone in a case of Hodgkin's disease. Acta Haematol 76: 178-180, 1986.

16. Weisenberg E, Anastasi J, Adeyanju M, Variakojis D and Vardiman JW: Hodgkin's disease associated with chronic lymphocytic leukemia. Eight additional cases, including two of the nodular lymphocyte predominant type. Am J Clin Pathol 103: 479-484, 1995.

17. Chan WC, Griem ML, Grozea PN, Freel RJ and Variakojis D: Mycosis fungoides and Hodgkin's disease occurring in the same patient. Report of three cases. Cancer 44: 1408-1413, 1979.

18. Harris NL: The relationship between Hodgkin's disease and non-Hodgkin's lymphoma. Semin Diagn Pathol 9: 304-310, 1992.
19. Loutayf Ranea JJ: Lymph node plasmacytoma as the $2 \mathrm{~d}$ tumor in a patient with Hodgkin's disease. Medicina (B Aires) 46: 252, 1986 (In Spanish)

20. Berkessy S, Radványi G, Nagy Z and Takács I: A case of multiple myeloma, associated with terminal plasma cell leukemia following recovery from Hodgkin's disease. Orv Hetil 129: 1053-1055, 1988 (In Hungarian).

21. Greenberg BB, Stats D and Goldberg M: The simultaneous occurrence of plasma cell multiple myeloma and Hodgkin's disease. N Y State J Med 50: 305-307, 1950.

22. Gelmann EP and Dennis LH: Plasma-cell dyscrasia after alkylating-agent therapy for Hodgkin's disease. N Engl J Med 305: 1350, 1981.

23. Hasskarl J, Ihorst G, De Pasquale D, Schröttner P, Zerweck A, Wäsch R and Engelhardt M: Association of multiple myeloma with different neoplasms: Systematic analysis in consecutive patients with myeloma. Leuk Lymphoma 52: 247-259, 2011.

24. Ibarrola de Andrés C, Toscano R, Lahuerta JJ and Martínez-González MA: Simultaneous occurrence of Hodgkin's disease, nodal Langerhans' cell histiocytosis and multiple myeloma IgA (kappa). Virchows Arch 434: 259-262, 1999.

25. Lalayanni C, Theodoridou S, Athanasiadou A, Saloum R and Tsatalas C: Simultaneous occurrence of multiple myeloma and Hodgkin's disease. A case report. Haematologica 85: 772-773, 2000 .

26. Sundeen J, Lipford E, Uppenkamp M, Sussman E, Wahl L, Raffeld $\mathrm{M}$ and Cossman J: Rearranged antigen receptor genes in Hodgkin's disease. Blood 70: 96-103, 1987.

27. Bräuninger A, Hansmann ML, Strickler JG, Dummer R, Burg G, Rajewsky $\mathrm{K}$ and Küppers R: Identification of common germinal center B-cell precursors in two patients with both Hodgkin's disease and non-Hodgkin's lymphoma. N Engl J Med 340: 1239-1247, 1999.

28. Ibbotson RM, Revell PA, Molland EA and Minton MJ: The simultaneous presence of Hodgkin's disease and myeloma. Postgrad Med J 53: 52-53, 1977.

29. Tilly H, Bastard C, Delastre T, Duval C, Bizet M, Lenormand B, Daucé JP, Monconduit $\mathrm{M}$ and Piguet H: Cytogenetic studies in untreated Hodgkin's disease. Blood 77: 1298-1304, 1991.

30. Lu ZY, Brailly H, Wijdenes J, Bataille R, Rossi JF and Klein B: Measurement of whole body interleukin-6 (IL-6) production: Prediction of the efficacy of anti-IL-6 treatments. Blood 86: 3123-3131, 1995.

31. Seymour JF, Talpaz M, Hagermeister FB, Cabanillas F and Kurzrock R: Clinical correlates of elevated serum levels of interleukin-6 in patients with untreated Hodgkin's disease. Am J Med 102: 21-28, 1997. 